\title{
ArguBlogging: An application for the Argument Web
}

\author{
Floris Bex ${ }^{\mathrm{a}, \mathrm{b}, *}$, Mark Snaith ${ }^{\mathrm{c}}$, John Lawrence ${ }^{\mathrm{c}}$, Chris Reed ${ }^{\mathrm{c}, 1}$ \\ ${ }^{\text {a }}$ University of Groningen, The Netherlands \\ ${ }^{\mathrm{b}}$ Utrecht University, The Netherlands \\ ${ }^{\mathrm{c}}$ University of Dundee, UK
}

\section{A R T I C L E I N F O}

\section{Article history:}

Received 27 July 2012

Received in revised form

10 January 2014

Accepted 18 February 2014

Available online 5 March 2014

\section{Keywords:}

Argumentation

Opinions

Blogging

Web Apps

\begin{abstract}
A B S T R A C T
In this paper, we present a software tool for 'ArguBlogging', which allows users to construct debate and discussions across blogs, linking existing and new online resources to form distributed, structured conversations. Arguments and counterarguments can be posed by giving opinions on one's own blog and replying to other bloggers' posts. The resulting argument structure is connected to the Argument Web, in which argumentative structures are made semantically explicit and machine-processable. We discuss the ArguBlogging tool and the underlying infrastructure and ontology of the Argument Web.
\end{abstract}

(c) 2014 Elsevier B.V. All rights reserved.

\section{Introduction}

Blogging has become commonplace in the online community as an open platform to share and discuss information and opinions. There are currently almost 60 million blogs on Tumblr alone ${ }^{2}$ and Blogspot/Blogger ${ }^{3}$ consistently ranks as one of the most visited sites on the Web. ${ }^{4}$ Bloggers comment on and argue about a huge variety of topics, from the newest video game to who should be the next president of the United States, and popular blogs often have large numbers of vocal followers who comment on the opinions both expressed in the blog and also by other commenters.

As such, the blogging phenomenon (and the Web as a whole) acts as an enabler of large-scale argumentation, where different views are constantly presented, challenged, and evaluated by a large and diverse user group. However, what is currently not captured is the explicit structure of argumentative viewpoints: opinions and discussions may be identified (or "tagged") by their topics, time, or participants but not the different facts, opinions,

\footnotetext{
* Corresponding author.

E-mail addresses: florisbex@gmail.com, f.j.bex@rug.nl (F. Bex), marksnaith@computing.dundee.ac.uk (M. Snaith) johnlawrence@computing.dundee.ac.uk (J. Lawrence), chris@computing.dundee.ac.uk (C. Reed).

1 School of Computing, University of Dundee, DD1 4HN, UK.

2 www.tumblr.com/about, 5-6-2012.

3 www.blogger.com.

4 www.google.com/adplanner/static/top1000/, 5-6-2012.
}

and arguments and how they relate to one another and, as such, contribute to the overall picture. Having such structure has the potential to enable far better visualisation, navigation and analysis of a debate, and makes it easier to automate support for the argumentation process, for example, by discovering inconsistencies among arguments or by discovering synergies among disputants.

In this paper, we present ${ }^{5}$ and evaluate a software tool for ArguBlogging, ${ }^{6}$ which allows users to blog with linked, semantically rich argument data. Arguments and counterarguments can be posed by giving opinions on one's own blog and replying to other bloggers' posts. The resulting argument structure is connected to the Argument Web, which is part of the Semantic Web [2]. Thus, these argument structures can be further viewed and manipulated using the different tools that work with the Argument Web [3]. Furthermore, by supporting explicit debate-like moves, ArguBlogging users are gently encouraged to better structure and organise their positions, thereby potentially raising the overall quality of argumentation online.

Below, we first introduce the ArguBlogging tool and the underlying Argument Web in an informal way, through some examples. In Section 4 we then look at the tool's architecture, underlying infrastructure and ontology in more detail, and we briefly discuss a user evaluation of the tool.

\footnotetext{
5 The system presented here is a newly implemented system loosely based on the earlier version presented in [1].

6 argublogging.com.
} 


\section{Introducing the Argument Web}

The Web is an ideal platform for enhancing critical debate, due to its ubiquity and openness. One of the drawbacks of existing technologies, such as forums, blogs, Twitter and so on is that these methods do not capture the structure of argumentative viewpoints explicitly. This makes the task of identifying, searching and evaluating the relationships among arguments difficult. Recently, a number of tools have begun to offer support for explicit argument and debate on the Web. [4] discuss a wide variety of tools for visualising, ${ }^{7}$ editing, ${ }^{8}$ annotating ${ }^{9}$ and searching ${ }^{10}$ arguments on the Web. However, these tools are all self-contained, and the arguments represented in them cannot be interchanged. For example, if one edits an argument in Debatepedia it is not possible to subsequently visualise it in ArguNet.

The approach proposed by the Argument Web [2,5,3] aims to provide infrastructure that supports argumentation in many different contexts and use cases by providing practical bridges between individual applications and application domains, and by offering a coherent ontology supported by a variety of search and display tools. Thus, with the Argument Web, the structure of arguments and debate is not captured on a separate, specifically designed website. Rather, this structure is URI-addressable: the Argument Web as a part of the Semantic Web, is offering a platform that combines linked argument data with software tools that make online debate intuitive for various audiences, including mediators, students, academics, broadcasters and bloggers. With the Argument Web we aim to build an open, extensible and re-useable infrastructure for large-scale argument representation, manipulation, and evaluation, allowing for structured argument and debate whilst at the same time tapping into the enormous user base of the World Wide Web.

One particularly topical issue in mid 2012 was the issue of Assad's Syria and the morality of potential Western intervention. ${ }^{11}$ We can use this topic in creating an example to illustrate the Argument Web. Say you search the Web for arguments by asking the question 'Should we invade Syria?'. You find a number of arguments with links to their sources: a video of a press conference in which the UK prime minister says that Assad should be held accountable for war crimes, a newspaper column claiming that Syria is different from Iraq, an article that explores the military feasibility of attacking Syria, and so on. You focus on the newspaper column, and this page links to other arguments posted on the Web that agree or disagree with the fact that 'Syria is different from Iraq'. One of the counterarguments, posted on a forum, states that the writer of the column is a 'leftie' who has no idea what the difference between Syria and Iraq is, or what real war is like for that matter. In the same forum, another user disagrees with this argument, stating that 'McCain is for intervention and he is not a leftie'.

In this way, the Argument Web makes it possible to follow a line of argument across disparate fora, user comments, professionally prepared editorials, multimedia resources and so on. Furthermore, at any time you can express your agreement or disagreement with one of the points made and post your own linked reply on your blog. Fig. 1 visualises the small 'web' of arguments from our example. Here, links tagged pro indicate that one statement agrees with another statement, that is, that one statement provides a reason

\footnotetext{
7 e.g. www.argunet.org.

8 e.g. www.debatepedia.com.

9 e.g. ova.computing.dundee.ac.uk.

10 e.g. www.discoursedb.org.

11 en.wikipedia.org/wiki/Syrian_civil_war.
}

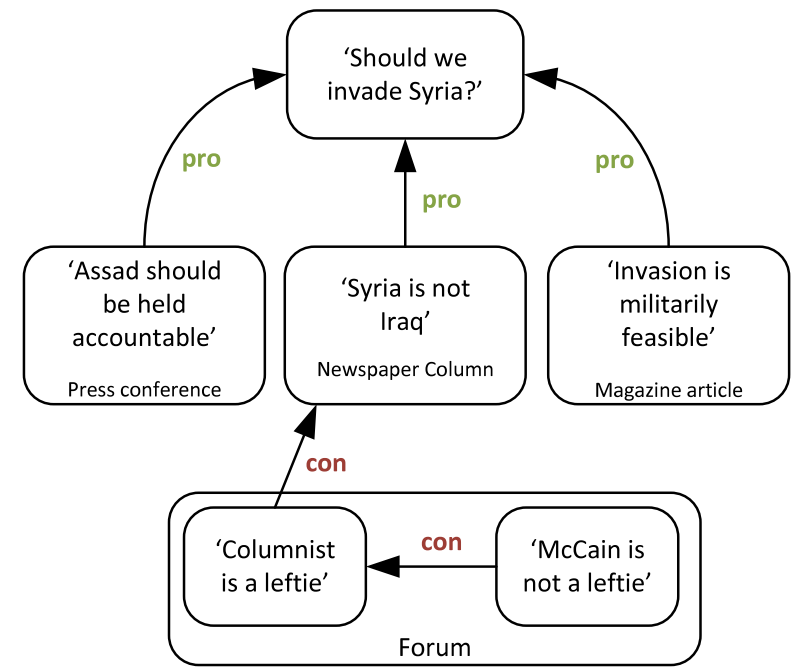

Fig. 1. Linked Argument Data across different online sources.

for the other statement; con links indicate that a counterargument is provided, that is, that one statement is a reason against another statement. Note that statements and arguments can be on different web pages but that, for example, a counterargument can also be given on the same web page (as is the case for the forum).

There are various tools for the Argument Web that all offer different interaction styles for engaging in online argument. ${ }^{12}$ For example, OVA ${ }^{13}$ can be used to annotate arguments on websites, the AIFdb ${ }^{14}$ interface can be used to search and visualise arguments, and TOAST $^{15}$ [3] can be used to evaluate arguments in the Argument Web according to a logical calculus [6]. It is further possible to upload and download argument graphs in formats associated with third-party argument visualisation and critical thinking tools such as Rationale ${ }^{16}$ and Carneades. ${ }^{17}$

\section{ArguBlogging}

Many of the existing tools for interfacing with the Argument Web are geared towards specialist audiences (e.g. academic users interested in discourse and argument analysis). The same is true for a lot of the tools discussed in [4]. With ArguBlogging the aim is to open up the Argument Web to regular internet users with a critical view: bloggers. The ArguBlogging tool is built so as to post arguments and link them to the Argument Web with minimal effort on the part of the user. The variety of Argument Web tools can then be used to further explore the more complex webs of opinions built using ArguBlogging and other tools.

The ArguBlogging tool requires no local installation, as it exists as a bookmarklet ${ }^{18}$ in the user's browser. Once the bookmarklet is installed from the ArguBlogging home page (argublogging.com), a user can respond to an opinion on a web page by highlighting the relevant piece of text and clicking the bookmarklet. The ArguBlogging widget is then rendered on the page, providing options with which to respond. In Fig. 2, the widget is rendered on top of an opinion column on a possible invasion of Syria.

\footnotetext{
12 www.argumentinterchange.org/library.

13 ova.computing.dundee.ac.uk/.

14 www.aifdb.org/.

15 www.arg.dundee.ac.uk/toast/.

16 rationale.austhink.com/.

17 carneades.github.com.

18 www.bookmarklets.com/about/.
} 


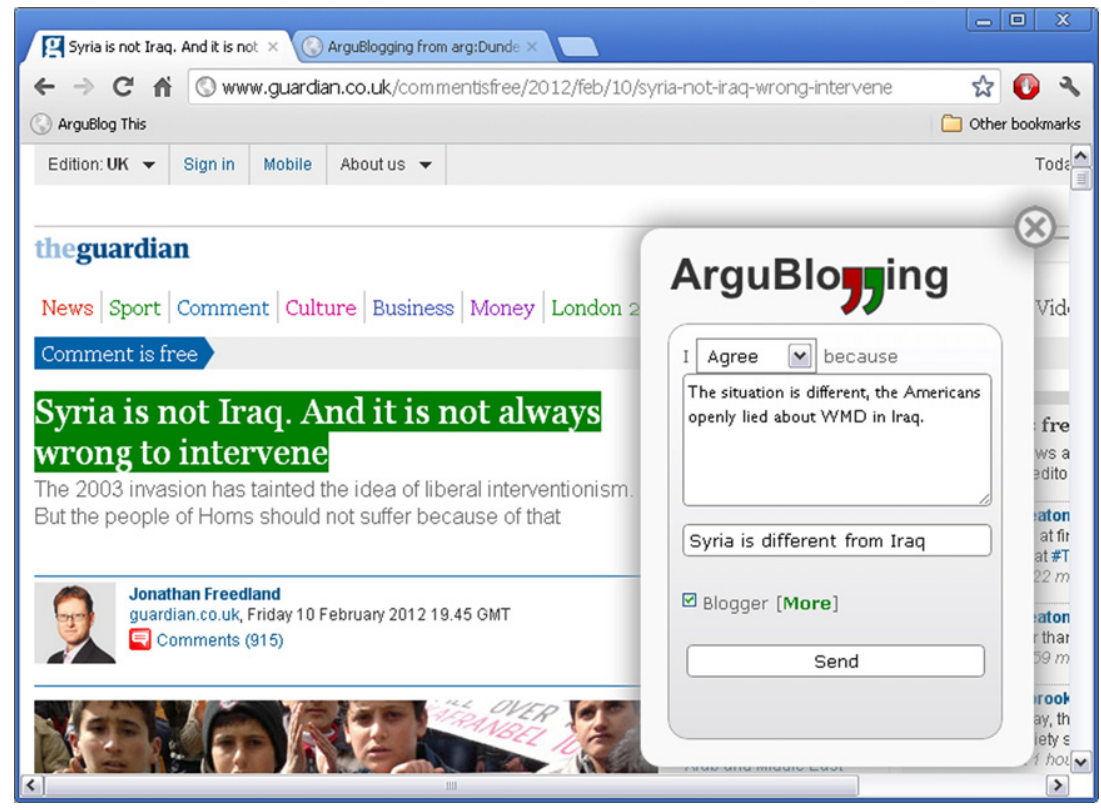

Fig. 2. ArguBlogging widget rendered on a web page.

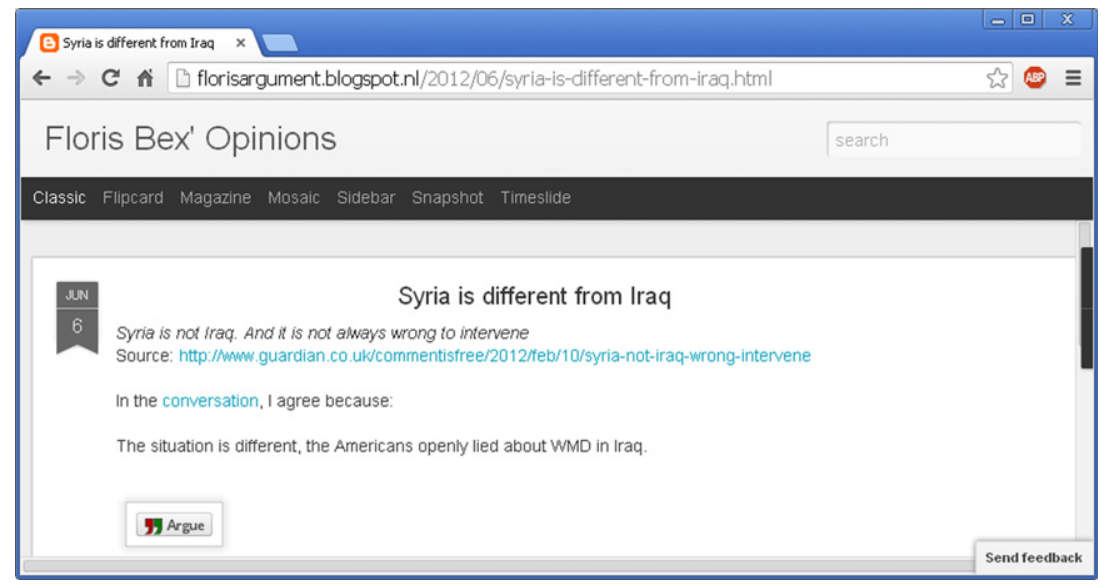

Fig. 3. A blog post automatically constructed by ArguBlogging

At the moment, the user can agree or disagree, causing the selected text to be highlighted in green or red, respectively. A large text field is available to give one's reasons for the agreement or disagreement; the smaller text field allows for the input of a title for the blog post. At the moment, the widget contains the ability to connect to two blogging platforms, Blogger and Tumblr.

Note that at the moment, the ArguBlogging tool uses a simple dialogue model. Currently, only agreement and disagreement can be expressed and it is not possible, for example, to challenge the other party to give a reason for her claim. The modular approach of the Argument Web means that other, more complex models of dialogue (see [7]) are easily integrated in the tools based on the Argument Web, including ArguBlogging. This future work is further discussed in Section 5.

Once the opinion is sent, the widget gives the user the links to the blog post and the conversation view. A typical blog post made using ArguBlogging is shown in Fig. 3.

Just below the title is the text that was replied to (in this case the text selected in the opinion column) and the link to the source. Below that is shown the blogger's conversational move (i.e. agree or disagree) together with the blogger's reasons for their opinion. There is also a link to the full conversation. At the bottom of the blog post an 'argue' button is created. This button is similar to the buttons which are often found on websites (e.g. 'share on Blogger', 'share on Facebook', 'share on Twitter'), as it allows one to respond to the blog post as a whole without having to select or copy the text in the blog. Clicking the button will bring up the ArguBlogging widget, and the text that is replied to is then the opinion of the blogger (in this case 'The situation is different, the Americans openly lied about WMD in Iraq.').

The ArguBlogging widget and blog posts created using ArguBlogging also provide a link to the conversation. This conversation includes all claims, opinions and arguments that are somehow relevant to (i.e. linked to) the opinion expressed in the blog post. Conversations can be viewed in the conversation view provided by the Argument Web search engine, ${ }^{19}$ which shows a sequence of replies as well as the argument that has been built in the conversation as a diagram. In Fig. 4, the conversation about whether Syria and Iraq are different is shown. ${ }^{20}$ On the left, opinions are rendered

\footnotetext{
19 www.AIFdb.org

20 www.arg.dundee.ac.uk/AIFdb/argview/750.
} 


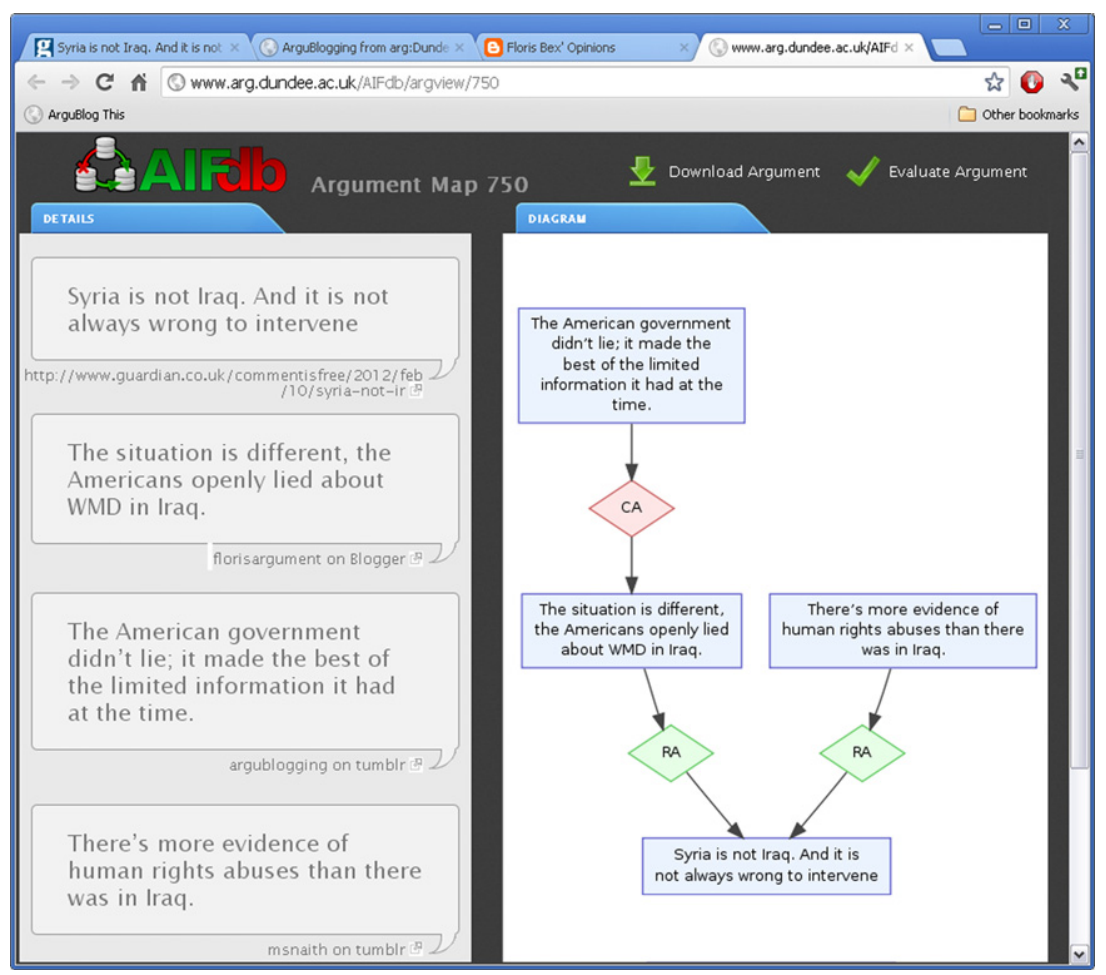

Fig. 4. The Argument Web search engine, showing the conversation on the left and the argument map on the right.

as a sequential dialogue between the participants in the conversation; below each speech bubble a link to the original source (opinion column, blog) is provided. On the right the argument based on this dialogue is rendered as a diagram. Here, green links represent pro links (reason for) and red links represent con links (reason against). The first reply discussed in our example - the blog post that America lied about WMD - is shown as a reason for the original text from the newspaper column. Other bloggers have also engaged in the debate, arguing against the statement that America lied and for the statement that Iraq is not Syria.

\section{Ontology, infrastructure and architecture}

Now that we have informally discussed ArguBlogging on the Argument Web, we offer a bit more detail with regards to the current underlying ontology and infrastructure of the Argument Web (Section 4.1) and the architecture of the ArguBlogging tool (Section 4.3).

\subsection{The AIF ontology}

The Argument Web is based on a common ontology for argument that supports web-based interchange of data, called the Argument Interchange Format (AIF) [5,6], which is available in a number of machine-readable formats (RDFs, OWL/XML, RDF/XML and SQL database schemas). ${ }^{21}$ The AIF ontology places at its core a distinction between information, such as propositions and sentences and schemes, general patterns of reasoning which, when applied, provide the specific argumentative relations. These schemes allow us to model and incorporate typical patterns of human argumentation [8], such as for example ad hominem argument, an argument against the person. Another important distinction in the ontology is that between argument structure, i.e. a static structure

21 www.arg.dundee.ac.uk/aif. of statements that are reasons for or against conclusions (as in, he prepared an argument), and argument dialogue, a debate or discussion (as in, they had an argument).

Fig. 5 shows an example of the linked data that corresponds to the discussion in our example. The locutions on the right are part of the dialogue in which one locution replies to another (shown in Fig. 4 as speech bubbles). The information on the left side of the picture (which was shown as a graph in Fig. 4) is the argument that follows from the dialogue. The dialogue and the argument are linked via nodes denoting the illocutionary force of the speech act in the locution, such as asserting or arguing. The illocutionary relation between the Reply node and the support node in the argument is more complex, as it represents the fact that it is in virtue of the fact that 'The situation is different' is responding to 'Syria is not Iraq' that there is an inferential support link here.

The detailed structure of linked data allows for the assessment of certain properties of argumentation that are not captured by other, simpler models (such as [9]). For example, in our model the felicity conditions of a speech act can be made explicit as conditions on the illocutionary force, and these conditions can then be subject to critical questioning and counterarguments. Thus, complex argumentative phenomena such as ad hominem argumentation or misquotation are easily captured and analysed.

\subsection{Argument Web infrastructure and architecture}

The Argument Web is a large-scale deployment of Semantic Web technology, which at the time of writing contained 1250 different argument maps (see Fig. 4) and more than 23,000 linked propositions (nodes). The Argument Web adopts a pragmatic approach to issues of infrastructure by specifying the ontology not only in OWL and RDF, but also as an instance of a relational database schema which can be implemented in a range of databases such as MySQL. Thus, the Argument Web approach builds on highly scalable, mature, robust and commercially accepted database systems, whilst still conforming to the main principles and demands of Linked Data: each piece of an argument 


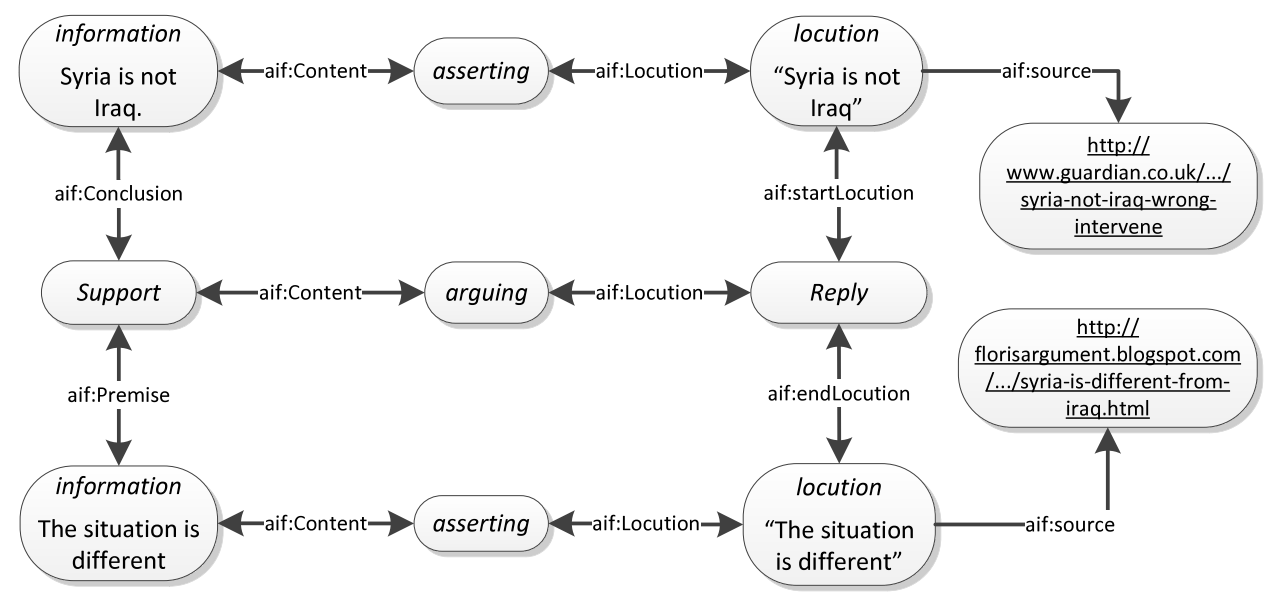

Fig. 5. Example of linked data on the Argument Web.

is addressable by a unique URI which stands in relationships to other URI-addressable information [10]. So an entity in the Argument Web can be accessed both by state-of-the-art Semantic Web technology (because it is expressed in RDF) and by a range of web services written in PHP which allow for the addition and retrieval of AIF components from the database to, for example, import and export argument data in a range of widely used formats including Araucaria (AML), DOT, Carneades (LKIF), JSON, Rationale (RTNL) and SVG files. Furthermore, a middle layer exists which groups simple web service queries, combining information about the structure and rules of argumentative dialogues with participant data so that more complex queries - for example, a query to determine what reasons a dialogue participant has for a particular point - can be performed.

\subsection{ArguBlogging architecture}

The ArguBlogging architecture (Fig. 6) consists of two main components: the user interface and the Argument Web Social Layer. The user interface (the widget) is a lightweight JavaScript application, accessed by a user from their web browser's bookmarks bar. Data from the bookmarklet is formatted as a JSON string and posted to the web service interface of the Argument Web Social Layer.

The Social Layer [11] provides an interface for applications to interact with both the Argument Web and social media sites. Data is received from applications via a web service, and is processed into both AIF and formats suitable for publishing to different social media platforms. The social layer also provides for user management, storing details of each registered user of the relevant tool (in this case ArguBlogging). Connections to the social media platforms are handled using the OAuth 2.0 protocol, allowing secure access to user blogs without the need for users to constantly provide their usernames and passwords.

\subsection{ArguBlogging user evaluation}

Because ArguBlogging is specifically targeted at non-academic end users we performed a relatively modest evaluation (15 users), asking respondents to rate and comment on the usability of the tool. For this evaluation, the users were asked to go to argublogging.com and install the bookmarklet. The users were then asked to respond to some opinion online using the ArguBlogging tool. Finally, the users could express their findings in a brief survey. ${ }^{22}$

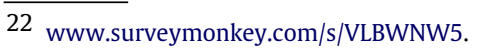
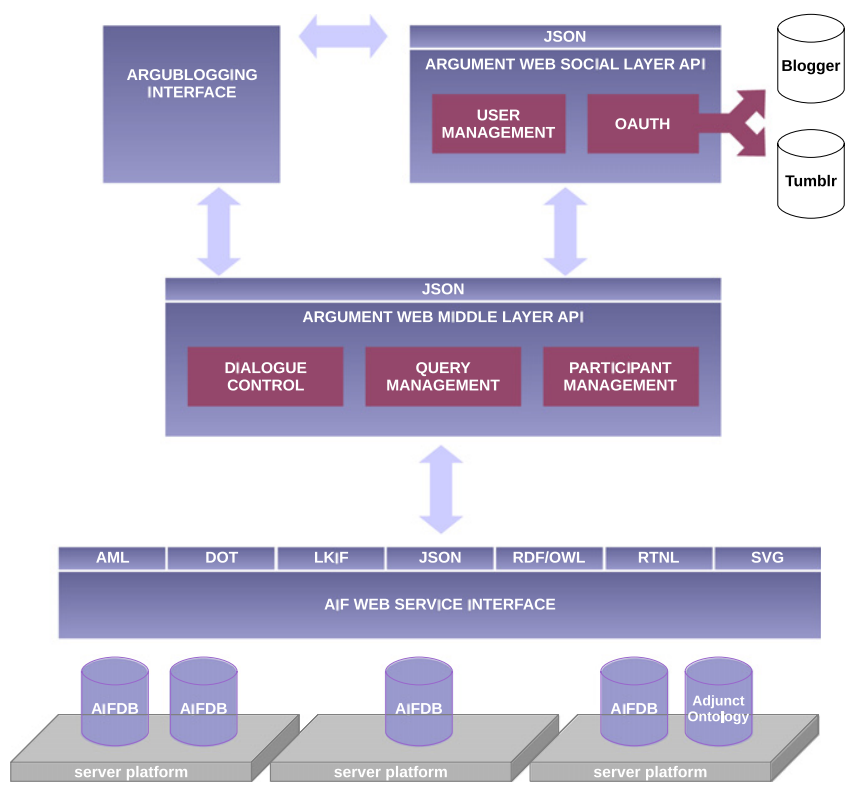

Fig. 6. The ArguBlogging architecture.

The survey starts by asking about the users' backgrounds. The users have quite a lot of experience with IT, with an average rating of 4.2 out of 5. Experience with argument and debate is less (average rating 2.8 out of 4 ), with half of the respondents only having argued in an informal setting and the other half having experience with debate in a formal setting (e.g. courtroom, academic). The experience with blogs is also average (rating 3 out of 5 ): about half of the users are bloggers or have blogged before and the other half has only read blogs; 1 user has never read or written for a blog. Most users have expressed opinions online before: stand-alone websites, forums, blogs and Twitter were all used in equal measure by various users. Two users indicated that they had never expressed an opinion online.

There are three questions in the survey which ask users to rate the difficulty of performing various actions with the ArguBlogging tool on a scale of 1-5: (Q1) Was it easy or difficult to configure Argublogging and get started?, (Q2) Were the Argublogging bookmarklet and window easy or difficult to use? and (Q3) Did you find it easy or difficult to view and respond to opinions after they had been posted? Table 1 shows the respondents' answers and the average scores. Respondents can further comment on the strength and weaknesses of ArguBlogging in open questions. 
Table 1

User scores for the usability of the ArguBlogging tool.

\begin{tabular}{lllllll}
\hline & Very difficult & Somewhat difficult & Neither difficult nor easy & Somewhat easy & Very easy & Avg. score (0-5) \\
\hline Q1 & $0(0 \%)$ & $5(33 \%)$ & $2(13 \%)$ & $2(13 \%)$ & $6(40 \%)$ & 3.6 \\
Q2 & $1(7 \%)$ & $3(20 \%)$ & $1(7 \%)$ & $4(27 \%)$ & $6(40 \%)$ & 3.73 \\
Q3 & $0(0 \%)$ & $2(13 \%)$ & $4(27 \%)$ & $6(40 \%)$ & $3(20 \%)$ & 3.67 \\
\hline
\end{tabular}

On average, the respondents found the tool relatively easy to use. The users almost consistently mentioned the simplicity and ease of use as one of the strengths of ArguBlogging. The difficulty of configuring ArguBlogging (Q1) was caused by four users not knowing what was meant by the "bookmarks toolbar" (the bar of favourite bookmarks under the browser's address bar). Other users commented that bookmarklets might not work well on smartphones and tablets; this can be remedied by developing an ArguBlogging app, which we leave for the future. The respondent who indicated 'very difficult' for Q2 did not use bookmarks and would have liked to be able to use ArguBlogging by copying text and a URL to the tool. Two respondents who indicated 'somewhat difficult' on Q1 and Q2 ran into technical problems which were related to the server, not the tool itself. This has probably influenced their ratings. Finally, with respect to Q3, users remarked that while the diagram (i.e. the conversation view, Fig. 4 ) is a nice feature, it is not very clear exactly what is represented.

When asked about the strengths and weaknesses of ArguBlogging, users were generally positive about ArguBlogging's simplicity and ability to react to any text online with a single click. Furthermore, they lauded the ability to link arguments across blogs and other media (news sites, forums), and the option to view connected arguments as a diagram was generally well-received. Not all users knew about or considered the wider implications of $\mathrm{Ar}$ guBlogging's connection to the Argument Web. One user indicated that it would be interesting to connect opinions across different blogs, and another user indicated that it would be useful to have discussions about one topic on a single page instead of scattered across different blogs; the Argument Web makes this possible, but one has to use the AIFdb search tool instead of ArguBlogging.

When asked about features they would like to see, the respondents indicated that the option to vote for or rate opinions (e.g. with a 'thumbs up' or 'thumbs down' button) would be nice. A few users would have liked to see Facebook and Twitter integration. This points to what about half of the users indicated as the major weakness of ArguBlogging, namely that it only posts to blogs. The reach of the tool and the wider Argument Web could be increased if similar tools are available to post to Twitter or social networking sites. A few users argued that such outlets would perhaps be more suited to the relatively short arguments posted with the simple ArguBlogging tool, as arguments on blogs tend to be more expansive and go on for several paragraphs.

\section{Conclusion and future work}

In this paper we have presented a Semantic Web approach to blogging that focuses on critical argument and debate, combining a rich ontology for describing argumentative and rhetorical structures with a flexible and extensible infrastructure for linked argument data.

By providing a simple bookmarklet and easy-to-use interface, structured and semantically rich data can be collected from users in an unobtrusive way. In this way, we aim to encourage the direct growth of the Argument and Semantic Web, as opposed to the common method of extracting structured semantic information from existing Web resources (DBpedia, ${ }^{23}$ for example, extracts its information from Wikipedia). Preliminary evaluation suggests that this approach to metadata collection is appealing, and user feedback suggests that it would be particularly appropriate to extend it to Argument Web applications for Twitter and Facebook.

ArguBlogging builds on a foundation for semantic blogging [12]. Hence, the connection argumentation and ontologies for social media, which was already explored in [9], can be easily made. The connection between blogs and semantically rich linked data opens up new research possibilities in, for example, areas like opinion mining and sentiment analysis. For example, if we combine the information on the Argument Web with information about social links (e.g. FAF $^{24}$ ), we can determine whether groups of people have the same opinions or agree with the same claims.

Currently, the ArguBlogging tool only allows for very simple argumentative discourse: given a claim, one can agree or disagree. Current research is working on generalised processing of formal dialogue systems [7], which provide rules for coherent dialogue that determine, for example, when each party takes its turn or which types of responses can be given to a claim or a question. In [13], we propose a generic framework for capturing these dialogue systems, which is explicitly compatible with the AIF ontology. Hence, dialogue systems expressed in the framework can be directly engineered into the tools that connect to the Argument Web, including ArguBlogging. On the one hand, this allows for a wider variety of argumentative moves (e.g. instead of disagreeing one can challenge the other blogger to give further reasons for his claim). On the other hand, this makes ArguBlogging not just a system for describing or capturing online argumentative discourse, but also for improving such discourse. The rules of a dialogue system, for example, might explicitly forbid attacks against the person such as the one in Section 4.1, allowing the debate to focus on what is really at stake. Thus, ArguBlogging will help to further the normative goal of the Argument Web, which is to bring a new kind of critical rationality to the Web.

\section{Acknowledgements}

This work has been supported in part by the EPSRC under grant $\mathrm{EP} / \mathrm{G} 060347 / 1$. The authors would also like to acknowledge the input and contributions from Simon Wells to earlier versions of the ArguBlogging system.

\section{References}

[1] S. Wells, C. Gourlay, C. Reed, Argument blogging. Working Notes of the 9th Int. Workshop on Computational Models of Natural Argument, CMNA 2009, Pasadena, 2009.

[2] I. Rahwan, Mass argumentation and the semantic web, Web Semantics 6 (1) (2008) 29-37.

[3] F. Bex, J. Lawrence, M. Snaith, C. Reed, Implementing the argument web, Commun. ACM 56 (10) (2013) 66-73.

[4] J. Schneider, T. Groza, A. Passant, A review of argumentation for the social semantic web, Semantic Web 4 (2) (2013) 159-218.

[5] I. Rahwan, F. Zablith, C. Reed, Laying the foundations for a world wide argument web, Artificial Intelligence 171 (10-15) (2007) 897-921.

[6] F. Bex, S. Modgil, H. Prakken, C. Reed, On logical reifications of the argument interchange format, J. Log. Comput. 23 (5) (2013) 951-989.

[7] P. Mcburney, S. Parsons, Dialogue Games for Agent Argumentation, in: I. Rahwan, G. Simari (Eds.), Argumentation in Artificial Intelligence, Springer, 2009, pp. 261-280. Chapter 22. 
[8] I. Rahwan, I. Banihashemi, C. Reed, D. Walton, S. Abdallah, Representing and classifying arguments on the semantic web, Knowl. Eng. Rev. 26 (4) (2011) 487-511.

9] C. Lange, U. Bojars, T. Groza, J. Breslin, S. Handschuh, Expressing argumentative discussions in social media sites. in: Social Data on the Web (SDoW2008), Workshop at the 7th Int. Semantic Web Conference, 2008.

[10] A. Hogan, J. Umbrich, A. Harth, R. Cyganiak, A. Polleres, S. Decker, An empirical survey of linked data conformance, J. Web Semantics 14 (2012) 14-44.
[11] M. Snaith, R. Medellin, J. Lawrence, C. Reed, Arguers and the argument web, in: Proceedings of CMNA 2010-2013, Lecture Notes in Artificial Intelligence, Springer, 2014, in press.

[12] S. Cayzer, Semantic blogging and decentralized knowledge management, Commun. ACM 47 (12) (2004) 47-52.

[13] F. Bex, C. Reed, Dialogue templates for automatic argument processing, in: Proceedings of COMMA 2012, Frontiers in Artificial Intelligence and Applications, vol. 245, IOS Press, 2012, pp. 266-377. 